\title{
Mining Corner Points on the Generic Shapes
}

\author{
M. Sarfraz ${ }^{1}$, Z. N. K. Swati ${ }^{2}$ \\ ${ }^{1}$ Department of Information Science, Kuwait University, Adailiya Campus, P.O. Box 5969, Safat 13060, Kuwait \\ Email: prof.m.sarfraz@gmail.com \\ ${ }^{2}$ Department of Computer Science and Information Technology, Karakoram International University, Gilgit-Baltistan, Pakistan \\ Email: zarnawab@kiu.edu.pk
}

Received 2012

\begin{abstract}
This paper designs and implements a corner detection algorithm for mining corner points on the generic shapes. The proposed corner detector detects corners by using combination of one rectangle and two ellipses (REE) with different parameter settings in their descriptions. REE combination slides along the boundary of the shape and records number of boundary points in each rectangle and ellipses. REE setup represents both local and global views of the image outline. The proposed technique presents a natural corners detection methodology to detect all true corners accurately. This technique is consistent with human vision system
\end{abstract}

Keywords: mining; corner points; corner detector; planar curves; generic shapes

\section{Introduction}

The extraction of corner points is useful for computer applications such as image recognition, segmentation, description, and data compression. Corner points play significant role in the area of computer graphics, pattern recognition, and computer vision. Corner points take part in document types object analysis such as map, chart, grid, table, and graph analysis. These points are used in multi-signal representation, shapes illustration, stereo system visualization, to track the movement of objects, image matching, construction of 2D mosaics [17], and the early phase of contour drawing systems. Many corner detection algorithms have been proposed in the existing literature [3-22].

Rosenfeld and Johnston [8] use k-cosine angle measurement to find corners. Rosenfeld and Weszka [9] introduce some modification of [8] in which the maxima of the difference between successive of the averaged $k$-cosine angle on a digital curve between the $k$ backward and forward vector were used. Freeman and Davis [6] corner detector contains the chain scanned [8] with line sliding along the contour. The change in local curvature is measured by calculating the difference in angles between consecutive segment locations and detects corners where highest change in curve occurs. The corner detector of Beus and Tiu [3] was modification of [6] which introduces an arm limit value $\tau$ to specify the upper bound for length of moving line.

The problem of corner detection can be divided into two types. First one is boundary-based approach and second one is region-based approach. This paper proposes a new corner detection algorithm using boundary-based approach. It is different from ordinary techniques $[3,5,6,8-16,20]$ which detect cosine angle for change in the boundary curve. The proposed algorithm defines its setting of geometry as a rectangle (R) and two ellipses (EE) for global, intermediate and local views respectively. Hence the setting can be denoted as REE which is different than EER which was defined in [23]. Like in $[15,16]$, it takes different scales/views (global, intermediate, local) of boundary curve with defined set of shapes (REE). Different parameters, in the description of REE determine global, intermediate, and local views of the boundary outline curve of an image. This combination is sliding along the planer curve and curve points are recorded at each move. Recorded curve points at each step are used for corner selection.

The proposed method REE and the method EER in [23] are using two ellipses and a rectangle. But the two methods are very different. In the proposed method, the rectangle used is outside the two ellipses whereas in [23], it is opposite. In addition to the new proposed method based on REE, the paper also makes a comparative study of four popular corner detection algorithms $[3,4,6,15]$ for boundary of shapes presented. The proposed technique is useful to detect corners from generic shape boundaries with or without noise.

The organization of the paper is done as follows. Section 2 gives basic formulation of the technique as well as the design of the proposed corner detection algorithm. Corner detection results are demonstrated in Section 3. A 
comparative study, with the existing algorithms in the current literature, is made in Section 4. The paper is finally concluded in Section 5.

\section{Proposed Algorithm}

Proposed technique of corner detection is based on rectangle $R$ and two ellipses $E_{1}$ and $E_{2}$ sliding along the given curve. $E_{1}$ and $E_{2}$ are embedded in $R$ such that $R \supset E_{1} \supset E_{2}$. The geometry of the rectangle and two ellipses is shown in Figure 1. To gather information about the locality of neighboring curve points, proposed technique uses rectangle and ellipses shown having common center at $p_{i}$.



Figure 1. Geometrical structure of NEW Algorithm.

The mathematical relations of $R$ and two ellipses $E_{1}$ and $E_{2}$ is described in Equation (1). The geometric structure of Figure 1 has been adopted as follows:

- The length and width of the rectangle $R$ are considered to be the lengths $2 A$ and $2 B$ respectively.

- The semi minor axis and semi major axis of the ellipse $E_{1}$ are considered to be the lengths $3 A / 4$ and $B$ respectively.

- The semi minor axis and semi major axis of the ellipse $E_{2}$ are considered to be the lengths $3 A / 4$ and $B / 2$ respectively.

$$
\left.\begin{array}{l}
R_{1}=2 A \times 2 B, \\
E_{1}=\pi \times 3 A / 4 \times B, \\
E_{2}=\pi \times 3 A / 4 \times B / 2, \\
\theta=\text { slope }^{\prime} S^{\prime}
\end{array}\right\}
$$

The length of rectangle and semi major axes of ellipses lie in the direction of slope ' $S$ '. Hence the width of rectangle and semi minor axes of ellipses lie at right angle to the slope ' $S$ ' of the contour with center at curve point $p_{i}, 1 \leq i \leq n$, where $n$ is the total number of contour points. Slope of curve point at $p_{i}$ is determined along the line drawn by calculating mean of five points (including $p_{i}$ ) on both sides of $p_{i}$.

By taking boundary point $p_{i}$ as center, the direction of rectangle $\mathrm{R}$ is adjusted along major axes of the Ellipses. Similarly, ellipses $E_{1}$ and $E_{2}$ having same center at $p_{i}$ are configured with same procedure. Thus $R \supset E_{1} \supset E_{2}$.



Figure 2. Snapshot of NEW Algorithm.

Combination of rectangle and ellipses slides on given curve and number of adjacent points for rectangle and each ellipse. It is recorded from $p_{i-A}$ to $p_{i+A}$ which lie in the area $R, E_{1}$, and $E_{2}$. Let $n R_{i}, n E_{1, i}$ and $n E_{2, i}$ describe the total number of curve points in rectangle $R$, ellipses $E_{1}$, and $E_{2}$ respectively, with centers at $i^{\text {th }}$ boundary point. For example in Figure $2, n R_{i}=23, n E_{1, i}=18$, and $n E_{2, i}=15$. Values of $n R_{i}, n E_{1, i}$ and $n E_{2, i}$, for each boundary point, are finally used while marking the absolute positions of corner points.

Proposed algorithm adopts natural corner detection methodology by combining three levels of views. Combination of one rectangle and two ellipses represents three special views of curve points. It traces number of counts $n R_{i}, n E_{1, i}$ and $n E_{2, i}$, for each boundary point. It calculates sufficient information to mark the absolute corners. Rectangle $R_{1}$ represents global view of boundary points and allowing only those boundary points for which $R_{1}-E_{1}=0$. These contour points are represented by set the $G$ as follows:

$$
\left.\begin{array}{l}
G=\left\{P=n R_{i}-n E_{1, i}=0\right\} \\
\text { Or } \\
G=\left\{P=n R_{i}-n E_{1, i}\right\}
\end{array}\right\}
$$

Set $G$ describes wider view of a shape and does not take false corners (at contour noise/irregularities). This is demonstrated in Figure 3 which shows some snapshots with curves noise/irregularity. Center points $p_{i}$ 's in Figures 3(a) and 3(b) look like corners if the local view of contour is taken, but if we observed the global view (broader part of the curve), these corners are rejected as they do not qualify the Equation (2). In general, $R_{1}-E_{1}=0$ pointing with arrows. Curve points in Figures 3(c) and 3(d) are only be considered in group $G$.

Curve points lying in the set $G$ are corner points in a relative way, they can be considered as candidate corner points. These points describe common region of contour where the actual corner is located as shown in the circled regions in Figure 4. Set $G$ describes connected points that represent a group and there is a possibility that more than one group may exist in set $G$. For each group, there is only single point which represents actual corner. The curve points in $E_{2, \mathrm{i}}$ of values $n E_{2, i}$ less than threshold ' $\eta$ ' are calculated for every group and smallest value $n E_{2, i}$ is 
selected as a corner. If in a group the number of points in $E_{2, i}$ are lower than ' $\eta$ ' then it means that corner point does not exist in that group. (a)

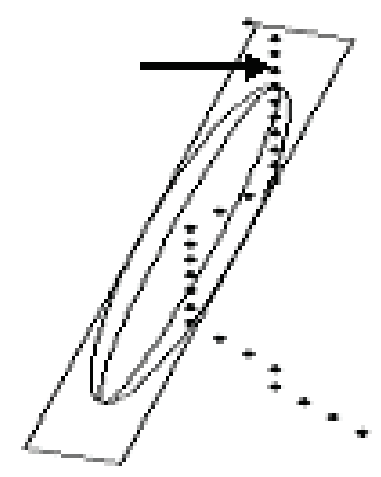

(b)

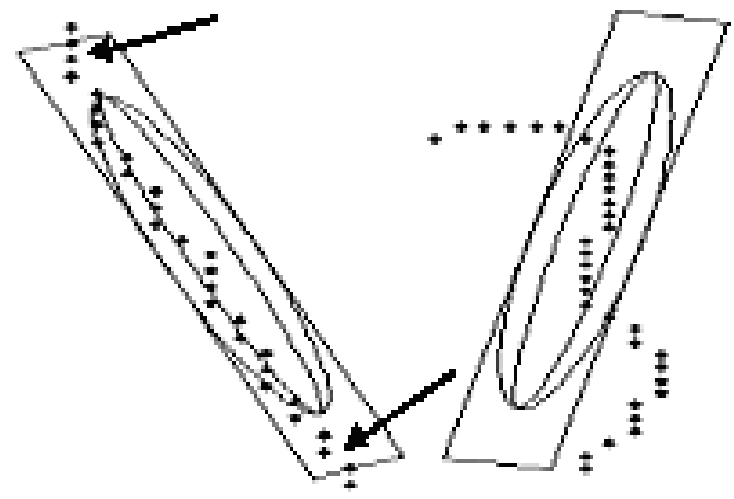

(d)

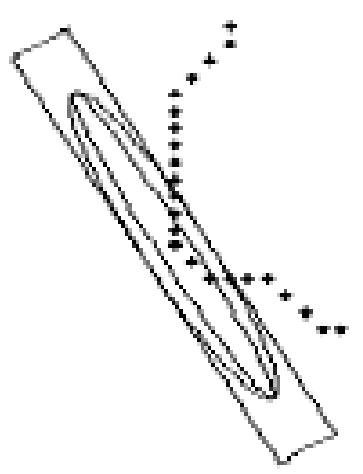

Figure 3. Some snapshots of NEW Algorithm for irregular boundary. (a), (b) are not qualified by $G$, and (c) is rejected due threshold ' $\eta$ '.

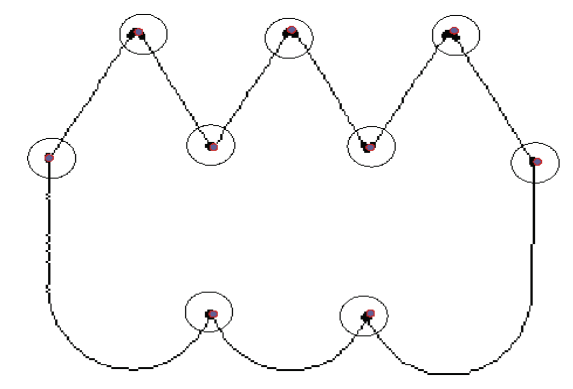

Figure 4. Some shapes marked (bold) with contour points in set $G$. Corners are marked in grey.

\section{Experimental Results}

To measure the accuracy of a corner detection algorithm, the absolute location of corner points must be known already. A board of 10 judges was used to mark the absolute position of corner points for standard shapes. Corner positions decided by majority were taken as absolute position of corners $[15,16]$.

These corner positions were used to judge the accuracy of different corner detection algorithms. Detected corners by proposed algorithm NEW are shown in Figure 8. One can see that the corner points, detected by NEW, have happened to be the same as detected by human eyes. It is important to note that this evaluation is based on Accuracy, localization error, noise sensitivity, transformation invariance, single response, parameter setting, and computation time.
For each contour point $P_{i}$

Count $n R_{i}, n E_{1, i}, n E_{2, i}$

End For

$G=\left\{P_{\mathrm{i}}: n R_{\mathrm{i}}=n E_{1, i}\right\}$

Make groups of connected points in $G$

For each group $G_{k^{*}}$

$$
\text { Corner }=\min _{n E_{2, j}}\left\{G_{k, j}: n E_{2, j}<\eta\right\}
$$

End For

Figure 5. Algorithm for proposed corner detector.

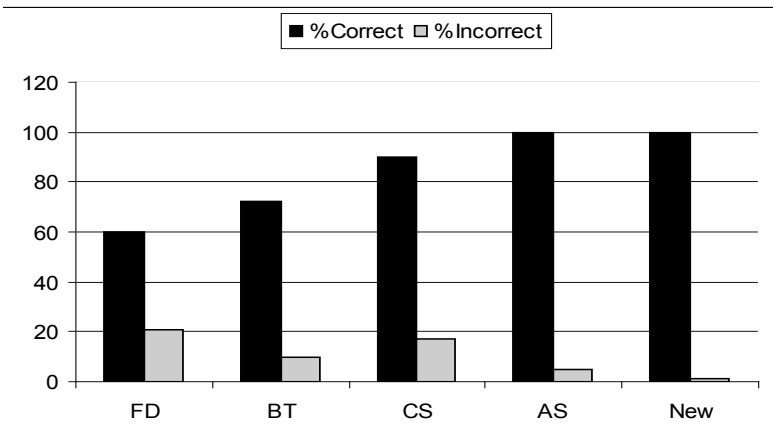

Figure 6. Result comparisons for each corner detector. 
M. Sarfraz ET AL.
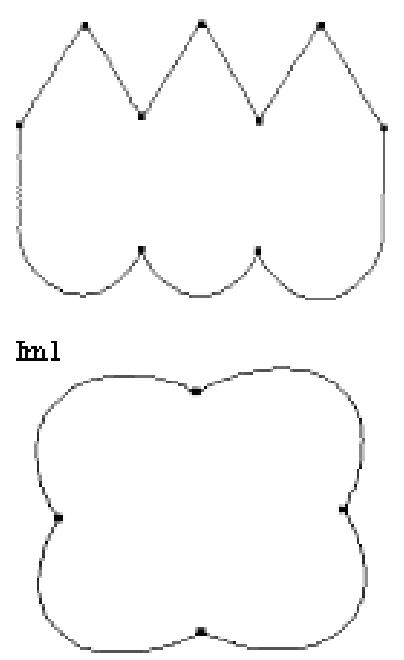

$\mathrm{Im} 3$

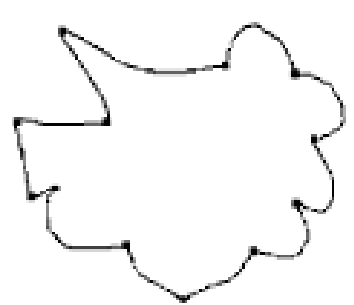

In 2

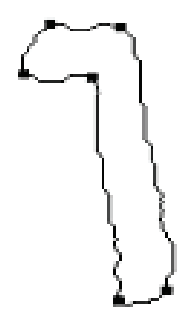

Im 4

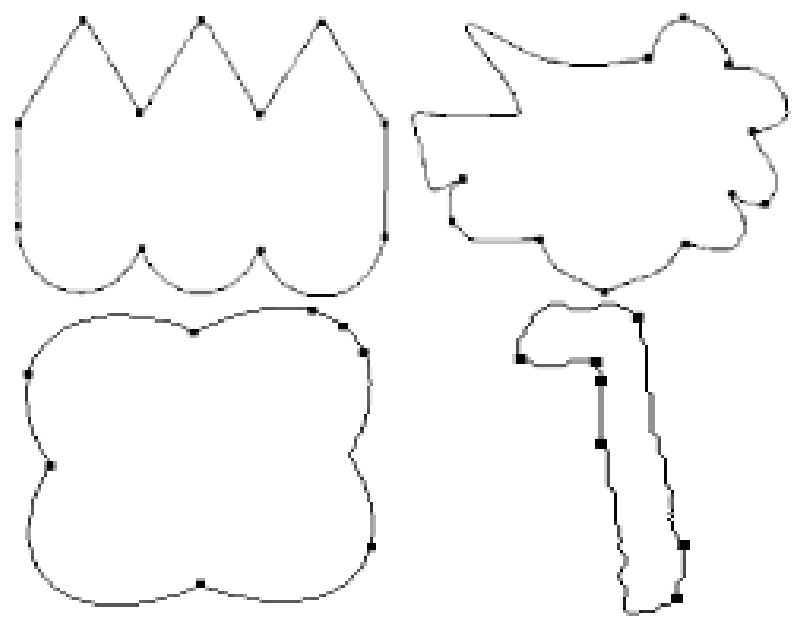

Figure 10. Corner detection by FD algorithm [6].

Figure 7. Test shapes with actual corner points [15].
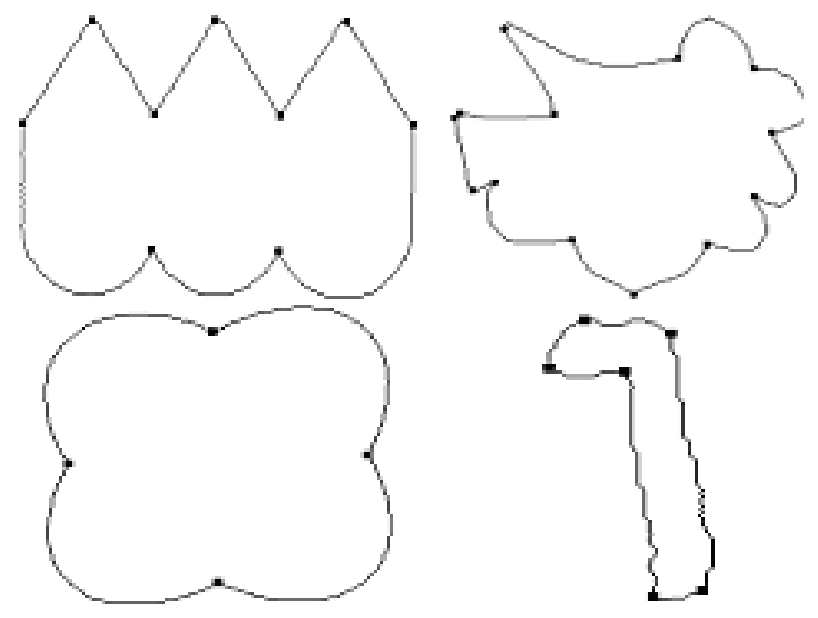

Figure 8. Corner detection by NEW Algorithm.
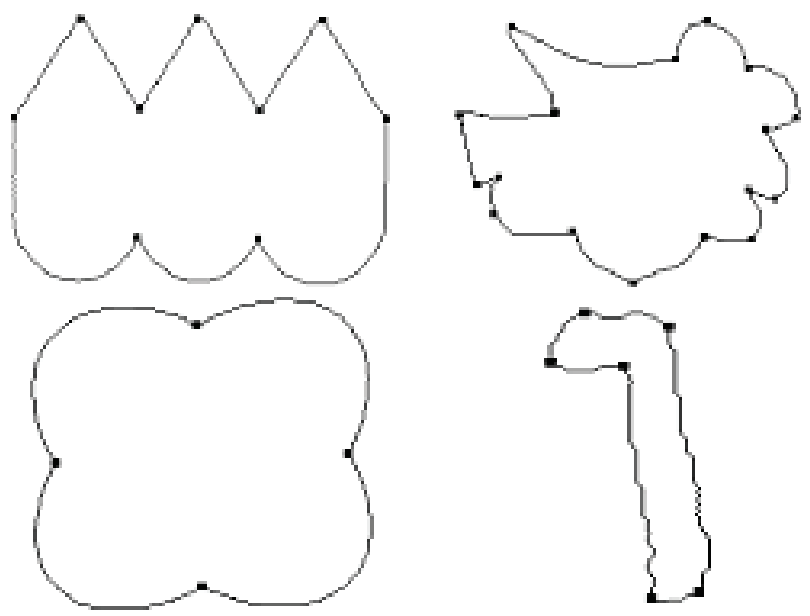

Figure 9. Corner detection by AS Algorithm [15].
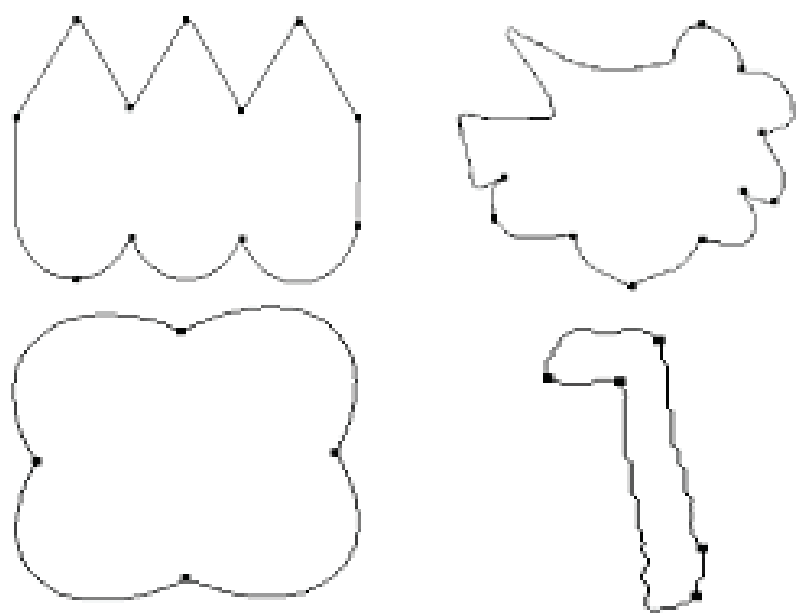

Figure 11. Corner detection by BT algorithm [3].
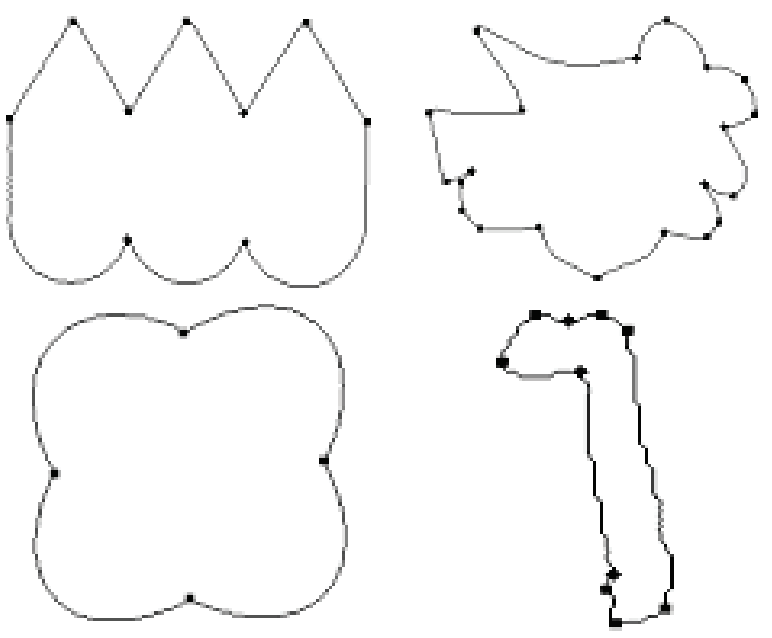

Figure 12. Corner detection by CS Algorithm [4]. 


\section{Comparative Study}

Different corner detection algorithms have been presented for digital boundary and their comparison has also been described by different authors. To evaluate a corner detection algorithm, it will properly be tested on variety of shapes. Four proposed Standard shapes, in Figure 7, contain noise/irregularities along the boundary curves, different types of varying curves, and angle sharpness. These types of variations are probable in real time shape contour. These test shapes are used in this paper for comparison/evaluation of different corner detectors. Four popular algorithms, namely BT[3], CS[4], FD[6], AS [15, 16] have been taken for comparison in our study. The proposed algorithm is referred as NEW.

A study of the five algorithms, in the light of the criteria mentioned in Section 3, has been made. Figure 8-12 shows the results of the five algorithms for the proposed test shapes in Figure 7 and their comparative graph is described in Figure 6.

Accuracy results for true corner points detected by FD corner detector were lowest $(60 \%)$ for standard shapes. It is clearly indicated (in im 3 and im4 of Figure 10) that FD corner detector ignores some true corners. Accurate corners marked by BT corner detector is $72 \%$ were better than previous corner detector, but it also tends to miss some important corners (in im4 of Figure 11). In case of incorrect corner detection and localization errors, the performance of BT is very good and is less than $10 \%$. CS [4] gives very good results in case of correct corner detection which is $90 \%$, but its false detection rate is high and is $17 \%$ which exceeds than BT. Accurate corners marked by AS corner detector are $100 \%$, but it also detects some false corner points (in im2 of Figure 9). Performance of this algorithm is better with respect to localization errors and false detections which is $5 \%$.



Figure 13. Corner detection by proposed Algorithm.

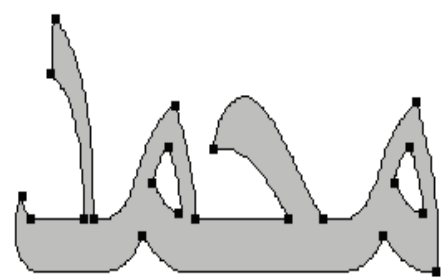

Figure 14. Corner detection by proposed Algorithm.
A considerable improvement can be seen in NEW that correctly marked $100 \%$ true corners. Lowest percentage of incorrect detection is $1 \%$ (in Figure 8) which is another big advantage of the proposed algorithm. One can hardly find localization error at any detected corner points. Performance of this algorithm must be appreciated in heavy noise conditions as well as complex shapes. No algorithm could accurately find all corners of im2, due to complex shape of the Figure. The proposed algorithm minimizes the incorrect corner(s) detection rate. Figures 13, 14, and 15 show corner detection on some more Shapes.

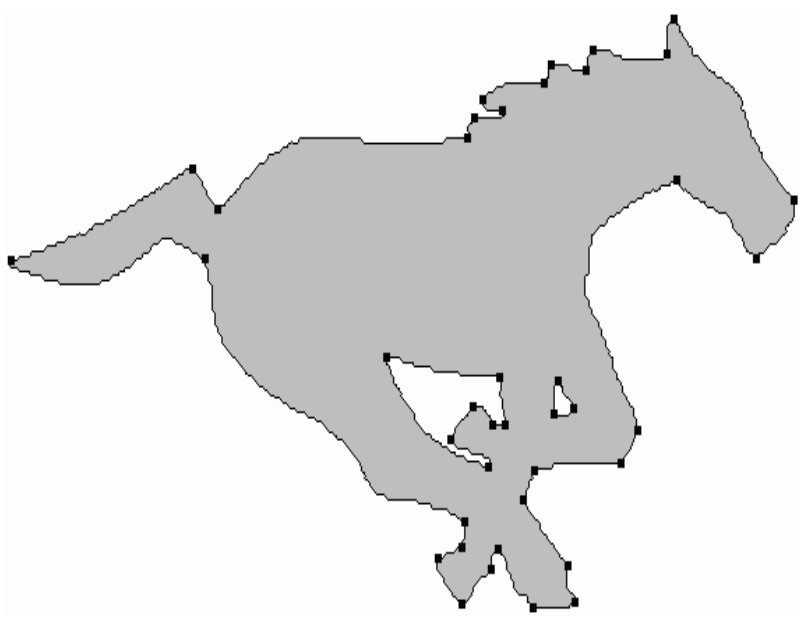

Figure 15. Corner detection by proposed Algorithm.

\section{Conclusion}

The new corner detection technique is proposed which does not involve curvature analysis and determination of cosine angle, hence making it very efficient. A comparative study of different corner detectors, based on proposed parameters, is given. Proposed corner detector has various advantages over previous corner detectors which are: (1) most consistent with human vision of corners; (2) ratio of false corner detection is extremely low; (3) very efficiently; (4) does not affect with transformation changes (5) highly insensitive to noise/irregularities along the curve; (6) robust to minor changes in size and resolution; and (7) very suitable for natural shapes/objects. Independent tuning of three parameters $(A, B$ and $\eta$ ) for optimum results, once set appropriately, is enough for most of the shapes.

There are various applications where corner detection algorithm can be used. Specifically in vectorizing outlines of images [1] and other curve manipulation applications [2], it is highly useful. The authors intend on such applications and use the proposed algorithm.

\section{Acknowledgements}

This work was supported by Kuwait University, Research Grant No. [WI 05/12]. 
M. Sarfraz ET AL.

\section{REFERENCES}

[1] M. Sarfraz, "Vectorizing Outlines of Generic Shapes by Cubic Spline using Simulated Annealing," International Journal of Computer Mathematics, Taylor \& Francis, Vol. 87, No. 8, 2010, pp. 1736 - 1751.

[2] M. Sarfraz, M. Z. Hussain and M. Hussain, "Shape Preserving Curve Interpolation," International Journal of Computer Mathematics, Taylor \& Francis, Vol. 89, No. 1, 2012 , pp. $35-53$.

[3] H. L. Beus and S. S. H. Tiu, "An Improved Corner Detection Algorithm based on Chain Coded Plane Curves," Pattern Recognition, Vol. 20, 1987, pp. 291-296.

[4] D. Chetverikov and Z. Szabo, "A Simple and Efficient Algorithm for Detection of High Curvature Points in Planner Curves," Proceedings of 23rd workshop of Australian Pattern Recognition Group, Steyr, 1999, pp.175-184.

[5] E. R. Davies, "Application of the generalized Hough transform to corner detection," Computers and Digital Techniques, IEE Proceedings E., Vol. 135, No. 1, 1988, pp. 49-54.

[6] H. Freeman and L. S. Davis, "A Corner Finding Algorithm for Chain-Coded Curves, " IEEE Trans. Computers, Vol. 26, 1977, pp. 297-303.

[7] H. C. Liu and L. S. Srinath, "Corner Detection from Chain-Code," Pattern Recognition, Vol. 23, 1990, pp. 51-68.

[8] A. Rosenfeld and E. Johnston, "Angle Detection on Digital Curves," IEEE Trans. Computers, Vol. 22, 1973, pp. 875-878.

[9] A. Rosenfeld and J. S. Weszka, "An Improved Method of Angle Detection on Digital Curves," IEEE Trans. Computers, Vol. 24, 1975, pp. 940-941.

[10] M. Sarfraz and M. R. Asim, A. Masood, "Capturing Outlines using Cubic Bezier Curves," Proc. of IEEE International Conference on Information \& Communication Technologies: from Theory to Applications, pp. 539 - 540, 2004.

[11] M. Sarfraz, A. Masood and M. R. Asim, "A Web Based System for Capturing Outlines of 2D Objects," Proc. of International Conference on Information \& Computer Science, Dhahran, Saudi Arabia, 2004.

[12] P. Smith, D. Sinclair, R. Cipolla and K. Wood, "Effective Corner Matching," In Paul H. Lewis, Mark S. Nixon, editors, Proc. 9th British Machine Vision Conference, Vol. II, 1998, pp. 545-556.
[13] S. Smith and J. Brady, "SUSAN - a new approach to low level image processing," Int.J.Comput.Vis., Vol. 23, 1995, pp.,45-78.

[14] C. Teh and R. Chin, "On the detection of dominant points on digital curves", IEEE Trans. PAMI, Vol. 8, 1990, pp. 859-873.

[15] A. Masood and M. Sarfraz, "A Novel Corner Detector Approach using Sliding Rectangles," The Proceedings of The 4th ACS/IEEE International Conference on Computer Systems and Applications (AICCSA-06), Sharjah, UAE, 2006, pp. 621 - 626, IEEE Computer Society Press.

[16] A. Masood and M. Sarfraz, "Corner Detection by Sliding Rectangles along Planar Curves," International Journal of Computers \& Graphics, Vol. 31, No. 3, 2007, pp. 440 -448 .

[17] I. Zoghlami, O. Faugeras and R. Deriche, "Using geometric corners to build a 2D mosaic from a set of images," Proceedings of the computer vision pattern recognition, 1997, p. 420-25.

[18] F. Attneave, "Some informational aspects of visual perception", Psychological Review, Vo. 61, 1954, pp. 183-93.

[19] A. Rattarangsi and R. T. Chin, "Scale-based detection of corners of planar curves," Transactions on Pattern Analysis and Machine Intelligence, Vol. 14, 1992, pp. 430-4.

[20] M. Sarfraz, A. Rasheed and Z. Muzaffar, "A Novel Linear Time Corner Detection Algorithm, Computer Graphics," Imaging and Visualization - New Trends, Sarfraz, M., Wang, Y., and Banissi, E. (Eds.), ISBN: 3-7695-2392-7, IEEE Computer Society, USA, 2005, pp. 191-196.

[21] L. Dreschler and H. H. Nagel, "On the selection of critical points and local curvature extrema of region boundaries for inter-frame matching," Proceedings of ICPR, 1982, pp. 542-44.

[22] A. J. Pritchard, S. J. Sangwine and R. E. N. Horne, "Corner and curve detection along a boundary using line segment triangles," Electronics Division Colloquium on Hough Transforms Digest, No. 1993/106, 1993, pp. 1-4.

[23] Z. N. K. Swati, S. Zaman, and M. Sarfraz, "A Novel Corner Detector Approach using Sliding two Ellipses and one Rectangle," The Proceedings of International Conference on Frontiers of Information Technology (FIT 2009), December 16-18, 2010, COMSATS Institute of Information Technology, Pakistan, Article \# 73, ISBN: 978-1-60558-642-7, ACM Press, 2010. 